\title{
Toxicology, Antidotes/Treatment
}

National Cancer Institute

\section{Source}

National Cancer Institute. Toxicology, Antidotes/Treatment. NCI Thesaurus. Code C19071.

The treatment or medication that can be administered to a person who has been exposed to a toxic substance 